\title{
Uncertainty analysis based on sensitivity applied to angle-ply composite structures
}

\author{
Carlos Conceição António, Luísa N. Hoffbauer
}

\begin{abstract}
This article describes a finite element-based formulation for the statistical analysis of the response of stochastic structural composite systems whose material properties are described by random fields. A first-order technique is used to obtain the second-order statistics for the structural response considering means and variances of the displacement and stress fields of plate or shell composite structures. Propagation of uncertainties depends on sensitivities taken as measurement of variation effects. The adjoint variable method is used to obtain the sensitivity matrix. This method is appropriated for composite structures due to the large number of random input parameters. Dominant effects on the stochastic characteristics are studied analyzing the influence of different random parameters. In particular, a study of the anisotropy influence on uncertainties propagation of angle-ply composites is carried out based on the proposed approach.
\end{abstract}

Keywords

Uncertainty propagation; Sensitivity analysis; First-order analysis; Angle-ply composites

\section{Introduction}

Uncertainty and sensitivity analysis are important parts of studying complex systems as composite laminated structures. Specifically, uncertainty analysis refers to the determination of the uncertainty in response results due to uncertainties in input parameters, and sensitivity analysis refers to the evaluation of the contributions of individual uncertainties in input parameters to the uncertainties in response results. The uncertainty under consideration can be classified as epistemic or aleatory. The epistemic uncertainty is often referred using alternative designations including state of knowledge, subjective and reducible. The epistemic uncertainty comes from a lack of knowledge of the appropriate value to consider for a quantity that is assumed to have a fixed value used in a particular analysis. Epistemic uncertainty is generally taken to be distinct from aleatory uncertainty under the conceptual and modeling point of view. Aleatory uncertainty arises from inherent randomness in the behavior of the system under study. Designations as variability, stochastic and irreducible are used for aleatory uncertainty. Several approaches to uncertainty and sensitivity analysis have been developed, including differential analysis, response surface methodology, Monte Carlo analysis, and variance decomposition procedures. Reviews of these methodologies are available in bibliography [1-7].

A probabilistic structural integrity analysis is needed due to deviations of the structural response of laminated composite structures produced by existing uncertainties in physical properties at the layer level. Nowadays the

definition of structural design criteria is based on ultimate state theory rather than on service stress theory. The

application of such concepts to composite materials based on reliability analysis creates new challenges to the

designer. A comprehensive review paper on this matter previous to 2002 is presented by Frangopol and Maute [8].

Optimal design of composite structures under probabilistic constraints is a very interesting field due to uncertainties associated with physical properties of fiber-reinforced composites [9-12]. Manufacturing of composite materials with fully specified profiles of materials is a complex 
process. Due to the large number of parameters involved, dispersions in microstructure, local volume fraction, lack of control quality, etc., the deviations may be inherent under the constraints of current fabrication technology. These fluctuations will reflect on the scattering of material properties, structural stiffness and consequently on the micro- and macro-mechanical behavior. Randomness of external loads can often be expected as well and it must be considered.

The problem of reliability-based design of laminated composite structures can be formulated as an optimization problem [8,9] or one can address the problem of alleviating the effects of unavoidable parameter uncertainties. Oh and Librescu [13] adopted this last strategy for free vibration of composite cantilevers under uncertainties at layer thickness, elastic constants and ply angle. The structural tailoring technique was applied to design laminated composite structures by searching the stacking sequence that corresponds to the less sensitive performance properties relatively to uncertainties in the input parameters.

In the present work the adopted strategy is similar to those proposed by $\mathrm{Oh}$ and Librescu [13] using different input parameters and applying static loading on laminated composite structures. Another objective of the proposed methodology is to identify the most important input parameters regarding the uncertainty propagation on structural response. This way the large number of input parameters involved in reliability analysis of laminated composite structures may be reduced.

This paper studies structural responses of statically loaded composite plate and shell structures with randomness in material properties. It is assumed that deviations of random parameters are not large with respect to their mean values. A first-order technique based on sensitivity analysis is therefore adopted handling the random scattering in multiple parameters system. A semi-analytical approach is used to obtain mean values and variances of displacement and the stress fields with known second-order statistics for various system input variables. In particular, the study of anisotropy influence on uncertainties propagation in angleply composites is carried out based on the proposed

approach.

\section{Uncertainty analysis based on sensitivity}

Sensitivity is a measure of the variation effect of a given input parameter on a required response. Usually the sensitivity is calculated as a first-order derivative of the response with respect to an input parameter. However, for certain nonlinear problems, higher order sensitivities are required. Relative sensitivity is related with the importance of the input parameter. The higher the relative sensitivity is, the more important the input parameter in question. Thus, one of the important aspects of sensitivity analysis is to identify the most important input parameters. Sensitivity analysis is also used for uncertainty analysis. The ingredients of the uncertainty analysis based on sensitivity methodology are the sensitivities and the covariance-variance matrix of the input parameters. Once these elements are available, uncertainty analysis based on sensitivity methodology is the most effective, straightforward approach [14].

In this work the quantification of response uncertainties of composite structures due to uncertainty in the properties and loads of the structural model is implemented based on linear statistical analysis. This methodology uses a Taylor's series expansion to obtain a linear relationship between the response random variables-displacements and stresses, and the random structural input parameters. The adjoint variable method is used to obtain the sensitivity matrix. This method is appropriated for composite structures due to the large number of random input parameters. No assumption is necessary concerning the form of the probability density function of the random input parameters. Knowledge of mean and covariance matrix for the random structural parameters plus structural response equations is all that is required to obtain the mean and covariance matrix of the response functions.

\section{Propagation of uncertainties}

In this work the calculated system response $\varphi$ is considered to be a real-valued function of $n$ system parameters denoted as $\mathbf{x}=\left(x_{1}, \ldots, x_{n}\right)$. The true values of these parameters are not known and so, only the nominal values $\mathbf{x}^{0}=\left(x_{1}^{0}, \ldots, x_{n}^{0}\right)$ and their uncertainties $\delta \mathbf{x}=$ $\left(\delta x_{1}, \ldots, \delta x_{k}\right)$ are available. Assuming the system parameters as random variables, the nominal values are taken to be the expected values and the associated uncertainties are given by the corresponding standard deviations. Commonly, the relative uncertainties $\delta x_{i} / x_{i}^{0}$ are symmetrically distributed in the neighborhood of $x_{i}^{0}$ and they are smaller than unity. The true parameter value is defined in vector form as

$\mathbf{x}=\mathbf{x}^{0}+\delta \mathbf{x}=\left(x_{1}^{0}+\delta x_{1}, \ldots, x_{n}^{0}+\delta x_{n}\right)$.

The response is related to the parameters using the equation of the computational model written in close form as

$\varphi=\varphi\left(x_{1}, \ldots, x_{n}\right)=\varphi\left(x_{1}^{0}+\delta x_{1}, \ldots, x_{n}^{0}+\delta x_{n}\right)$.

In the above functional relationship $\varphi$ is used in both senses as random function and as its numerical realization. The expansion in Taylor's series of functional (2) around the nominal value $\mathbf{x}^{0}=\left(x_{1}^{0}, \ldots, x_{n}^{0}\right)$ considering only the terms up to the $n$th order is the following:

$$
\begin{aligned}
\varphi\left(x_{1}, \ldots, x_{n}\right) & =\varphi\left(x_{1}^{0}+\delta x_{1}, \ldots, x_{n}^{0}+\delta x_{n}\right) \\
& =\varphi\left(x_{1}^{0}, \ldots, x_{n}^{0}\right)+\sum_{i_{1}=1}^{n}\left(\frac{\partial \varphi}{\partial x_{i_{1}}}\right)_{\mathbf{x}^{0}}\left(x_{i_{1}}-x_{i_{1}}^{0}\right)
\end{aligned}
$$




$$
\begin{aligned}
& +\frac{1}{2} \sum_{i_{1}, i_{2}=1}^{n}\left(\frac{\partial^{2} \varphi}{\partial x_{i_{1}} \partial x_{i_{2}}}\right)_{\mathbf{x}^{0}}\left(x_{i_{1}}-x_{i_{1}}^{0}\right)\left(x_{i_{2}}-x_{i_{2}}^{0}\right) \\
& +\frac{1}{3 !} \sum_{i_{1}, i_{2}, i_{5}=1}^{n}\left(\frac{\partial^{3} \varphi}{\partial x_{i_{1}} \partial x_{i_{2}} \partial x_{i_{3}}}\right)_{\mathbf{x}^{0}} \\
& \times\left(x_{i_{1}}-x_{i_{1}}^{0}\right)\left(x_{i_{2}}-x_{i_{2}}^{0}\right)\left(x_{i_{5}}-x_{i_{3}}^{0}\right)+\ldots \\
& +\frac{1}{n !} \sum_{i_{1}, i_{2}, \ldots, i_{n}=1}^{n}\left(\frac{\partial^{n} \varphi}{\partial x_{i_{1}} \partial x_{i_{2}} \ldots \partial x_{i_{n}}}\right)_{\mathbf{x}^{0}} \\
& \times\left(x_{i_{1}}-x_{i_{1}}^{0}\right)\left(x_{i_{2}}-x_{i_{2}}^{0}\right) \ldots\left(x_{i_{n}}-x_{i_{n}}^{0}\right)
\end{aligned}
$$

and using relationship (2) it gives

$$
\begin{aligned}
\varphi\left(x_{1}, \ldots, x_{n}\right)= & \varphi\left(x_{1}^{0}+\delta x_{1}, \ldots, x_{n}^{0}+\delta x_{n}\right) \\
= & \varphi\left(x_{1}^{0}, \ldots, x_{n}^{0}\right)+\sum_{i_{1}=1}^{n}\left(\frac{\partial \varphi}{\partial x_{i_{1}}}\right)_{\mathbf{x}^{0}} \delta x_{i_{1}} \\
& +\frac{1}{2} \sum_{i_{1}, i_{2}=1}^{n}\left(\frac{\partial^{2} \varphi}{\partial x_{i_{1}} \partial x_{i_{2}}}\right)_{\mathbf{x}^{0}} \delta x_{i_{1}} \delta x_{i_{2}} \\
& +\frac{1}{3 !} \sum_{i_{1}, i_{2}, i_{5}=1}^{n}\left(\frac{\partial^{3} \varphi}{\partial x_{i_{1}} \partial x_{i_{2}} \partial x_{i_{3}}}\right)_{\mathbf{x}^{0}} \delta x_{i_{1}} \delta x_{i_{2}} \delta x_{i_{5}} \\
& +\ldots+\frac{1}{n !} \sum_{i_{1}, i_{2}, \ldots, i_{n}=1}^{n}\left(\frac{\partial^{n} \varphi}{\partial x_{i_{1}} \partial x_{i_{2}} \ldots \partial x_{i_{n}}}\right)_{\mathbf{x}^{0}} \\
& \times \delta x_{i_{1}} \delta x_{i_{2}} \ldots \delta x_{i_{n}} .
\end{aligned}
$$

Different moments as mean, variance, skewness and kurtosis of the random variable $\varphi\left(x_{1}, \ldots, x_{n}\right)$ can be obtained from Eq. (4) considering that the system parameters are random variables with a joint probability density function $\Phi\left(x_{1}, \ldots, x_{n}\right)$.

The use of nonlinear terms in (4) is impracticable for large complex systems with many parameters. Then, the linear expansion of the response $\varphi\left(x_{1}, \ldots, x_{n}\right)$ is used as follows

$$
\begin{aligned}
\varphi\left(x_{1}, \ldots, x_{n}\right) & =\varphi\left(\mathbf{x}^{0}\right)+\sum_{i=1}^{n}\left(\frac{\partial \varphi}{\partial x_{i}}\right)_{\mathbf{x}^{0}} \delta x_{i} \\
& =\varphi^{0}+\sum_{i=1}^{n} S_{i} \delta x_{i}
\end{aligned}
$$

being $\varphi^{0} \equiv \varphi\left(\mathbf{x}^{0}\right)$ and $S_{i}=\left(\partial \varphi / \partial x_{i}\right)_{\mathbf{x}^{0}}$ the response sensitivity to parameter $x_{i}$. The mean value and the variance of the response is obtained respectively from (5) as

$$
E(\varphi) \equiv \varphi^{0},
$$

$$
\begin{aligned}
\operatorname{var}(\varphi) \equiv & E\left(\left(\varphi-\varphi^{0}\right)^{2}\right)=\sum_{i=1}^{n} S_{i}^{2} \operatorname{var}\left(x_{i}\right) \\
& +2 \sum_{i \neq j=1}^{n} S_{i} S_{j} \operatorname{cov}\left(x_{i}, x_{j}\right)
\end{aligned}
$$

The last equation can be written in matrix form as

$\operatorname{var}(\varphi)=\mathbf{S C}_{x} \mathbf{S}^{\mathbf{T}}$,

where the superscript " $\mathrm{T}$ " denotes the transposition, $\mathbf{C}_{x}$ is the covariance matrix for parameters $\left(x_{1}, \ldots, x_{n}\right)$ with components defined as

$\left(\mathbf{C}_{x}\right)_{i j}= \begin{cases}\operatorname{cov}\left(x_{i}, x_{j}\right)=\rho_{i j} \sigma_{i} \sigma_{j}, & i \neq j, \\ \rho_{i j} \equiv \text { correlation coefficient } & \\ \operatorname{var}\left(x_{i}\right)=\sigma^{2}, & i=j\end{cases}$

and the column vector $\mathbf{S}=\left(S_{1}, \ldots, S_{n}\right)$ has components $S_{i}=\left(\partial \varphi / \partial x_{i}\right)_{x^{0}}$.

If the system parameters $\left(x_{1}, \ldots, x_{n}\right)$ are uncorrelated then Eq. (7) can be reduced to

$\operatorname{var}(\varphi)=\sum_{i=1}^{n} S_{i}^{2} \operatorname{var}\left(x_{i}\right)=\sum_{i=1}^{n} S_{i}^{2} \sigma_{i}^{2}$.

The previous concepts can be extended to the case of $m$ response functions all of them depending on parameters $\left(x_{1}, \ldots, x_{n}\right)$. Firstly, considering vector notation the $m$ responses can be presented as

$\boldsymbol{\varphi}=\left(\varphi_{1}, \ldots, \varphi_{m}\right)$

and the corresponding equivalent equations to $\mathrm{Eq}$. (5) are the following first-order Taylor expansion of $\varphi=(\mathbf{x})$ :

$$
\begin{aligned}
\varphi\left(\mathbf{x}^{0}+\delta \mathbf{x}\right) & =\varphi\left(\mathbf{x}^{0}\right)+\delta \varphi \\
& \simeq \varphi\left(\mathbf{x}^{0}\right)+\mathbf{S} \delta \mathbf{x},
\end{aligned}
$$

where $\mathrm{S}$ is a rectangular matrix of order $m \times n$ with components representing the sensitivity of the $j$ th response to the $i$ th system parameter such as

$(\mathbf{S})_{j}=\partial \varphi_{j} / \partial x_{i}$.

The expectation $E(\varphi)$ of $\varphi$ is obtained using the same procedure adapted to Eq. (6):

$E(\varphi)=\varphi^{0}$

Finally the covariance matrix $\mathrm{C}_{\varphi}$ for $\varphi$ is obtained by a similar procedure applied to Eq. (8) and this is

$$
\begin{aligned}
\mathbf{C}_{\mathbf{Q}} & =E\left(\mathbf{S} \delta \mathbf{x}(\mathbf{S} \delta \mathbf{x})^{\mathrm{T}}\right)=\mathbf{S} E\left(\delta \mathbf{x} \delta \mathbf{x}^{\mathrm{T}}\right) \mathbf{S}^{\mathrm{T}} \\
& =\mathbf{S C}_{x} \mathbf{S}^{\mathrm{T}}
\end{aligned}
$$

Equations for the propagation of higher order moments become very complex and are avoided in practice. From Eq. (15) for the propagation of uncertainties dependence of the covariance matrix $C_{\varphi}$ relatively to the sensitivity matrix $\mathbf{S}$ is observed. The components of this matrix are evaluated using the adjoint sensitivity analysis procedure.

\section{The adjoint sensitivity analysis procedure}

The objective of sensitivity analysis is to analyze the behavior of the response of the system and to evaluate the sensitivities of the system response to variations in the system input parameters around their nominal values. The methodology presented here is based on the adjoint variable method.

In this work the linear behavior of structural systems with the equilibrium equation set established is 
considered as

$\mathbf{K}(\mathbf{x}) \mathbf{u}=\mathbf{F}$,

where $\mathbf{K}$ is the stiffness matrix, $\mathbf{u}$ is the displacement vector and $\mathbf{F}$ are the applied external loads.

In the adjoint variable method, an augmented Lagrangian is defined in terms of adjoint variable fields in order to eliminate the implicit derivatives. Following the method proposed by Arora and Cardoso [15], considering a given functional $\varphi=\varphi\left(x_{1}, \ldots, x_{n}\right)$ and writing the response equation of the system in the following form

$\Psi(\mathbf{u}, \mathbf{x})=\mathbf{K}(\mathbf{x}) \mathbf{u}-\mathbf{F}$

the augmented functional can be written as

$L(\mathbf{u}, \mathbf{x}, \boldsymbol{\phi})=\varphi(\mathbf{u}, \mathbf{x})-\boldsymbol{\phi}^{T} \mathbf{\Psi}(\mathbf{u}, \mathbf{x})$.

The vector of adjoint variables $\phi$ is assumed as Lagrange multipliers selected to make stationary the functional $L$ relatively to the displacement vector $\mathbf{u}$. This condition can be formulated as

$\frac{\partial L}{\partial \mathbf{u}}=\frac{\partial \varphi(\mathbf{u}, \mathbf{x})}{\partial \mathbf{u}}-\phi^{\mathrm{T}} \frac{\partial \boldsymbol{\Psi}(\mathbf{u}, \mathbf{x})}{\partial \mathbf{u}}=\mathbf{0}$.

Considering the independence of $\mathbf{F}$ to the displacements $\mathbf{u}$ and Eq. (17), the adjoint set of equations is obtained

$\mathbf{K}(\mathbf{x}) \boldsymbol{\phi}=\frac{\partial \varphi(\mathbf{u}, \mathbf{x})}{\partial \mathbf{u}}$

being the tangent stiffness matrix defined for the equilibrium solution

$\Psi(\mathbf{u}, \mathbf{x})=\mathbf{K}(\mathbf{x}) \mathbf{u}-\mathbf{F}=0$.

On the other hand taking into account that in an equilibrium situation the functional (17) is stationary, it proofs [15] that

$\frac{\mathrm{d} \varphi}{\mathrm{d} \mathbf{x}}=\frac{\partial L}{\partial \mathbf{x}}$.

Differentiating (18) to variables $\mathbf{x}$ it is obtained

$$
\begin{aligned}
\frac{\partial L}{\partial \mathbf{x}}= & \frac{\partial \varphi(\mathbf{u}, \mathbf{x})}{\partial \mathbf{x}}+\frac{\partial \varphi(\mathbf{u}, \mathbf{x})}{\partial \mathbf{u}} \frac{\partial \mathbf{u}}{\partial \mathbf{x}} \\
& -\boldsymbol{\phi}^{\mathbf{T}}\left[\frac{\partial \boldsymbol{\Psi}(\mathbf{u}, \mathbf{x})}{\partial \mathbf{x}}+\frac{\partial \boldsymbol{\Psi}(\mathbf{u}, \mathbf{x})}{\partial \mathbf{u}} \frac{\partial \mathbf{u}}{\partial \mathbf{x}}\right]
\end{aligned}
$$

that can be simplified using equality (19) yielding to

$\frac{\partial L}{\partial \mathbf{x}}=\frac{\partial \varphi(\mathbf{u}, \mathbf{x})}{\partial \mathbf{x}}-\phi^{\mathbf{T}} \frac{\partial \boldsymbol{\Psi}(\mathbf{u}, \mathbf{x})}{\partial \mathbf{x}}$.

Considering the independence of $\mathbf{F}$ to variables $\mathbf{x}$ and using Eq. (17) it gives

$\frac{\mathrm{d} \varphi}{\mathrm{d} x}=\frac{\partial L}{\partial \mathbf{x}}=\frac{\partial \varphi(\mathbf{u}, \mathbf{x})}{\partial \mathbf{x}}-\phi^{\mathrm{T}} \frac{\partial \mathbf{K}(\mathbf{x})}{\partial \mathbf{x}} \mathbf{u}$.

The adopted methodology for sensitivity analysis is twofold:

First: Solve the adjoint set of Eq. (20);

Second: Get the sensitivities from Eq. (25).

\section{Applications to composite structures}

The previously described uncertainty analysis based on sensitivity has been applied to composite structures. The type of structure studied in this work considers a laminate shell structure commonly used in composite materials applications. The structural analysis is carried out using a displacement formulation of the Finite Element Method. The adopted element is the three-dimensional degenerated shell element with material anisotropy and stacking sequence of layers developed by Ahmad [16]. It is an isoparametric element with eight nodes and five freedom degrees per node based on the Mindlin shell theory. A structural application to angle-ply composite laminates is carried out using this approach.

\subsection{Problem definition}

The cylindrical shell laminate structure considered in this work is shown in Fig. 1. Considering material and geometric symmetries half the structure is taken. The half structure is divided into four macro-elements grouping all elements. There is one laminate per macro-element. Balanced $\pm \theta$ angle-ply laminates with five layers are considered. Each laminate has the same properties namely thickness, number of layers and stacking sequence of ply angles and materials. Thickness continuity is imposed on the macro-elements boundaries.

The cylindrical shell is hinged on linear sides and free on curvilinear ones. A set of discrete vertical loads varying from $P_{\max }$ to $P_{\min }$ is applied along the edge defined by the symmetry plane of the structure.

The mean values of elastic and strength properties of the ply material used in the laminate construction of the composite structure are presented in Table 1. The elastic constants of orthotropic ply are the longitudinal elastic modulus $E_{1}$, the transversal elastic modulus $E_{2}$, the inplane shear modulus $G_{1,2}$, the out-of-plane shear modulus $G_{1,3}$ and $G_{2,3}$, the in-plane Poisson's ratio $v_{1,2}$ and the outof-plane Poisson's ratios $v_{1,3}$ and $v_{2,3}$. The ply strength properties are the longitudinal strength in tensile $X$ and in compression $X^{\prime}$, the transversal strength in tensile $Y$ and in compression $Y^{\prime}$, and the shear strength $S$. The ply material

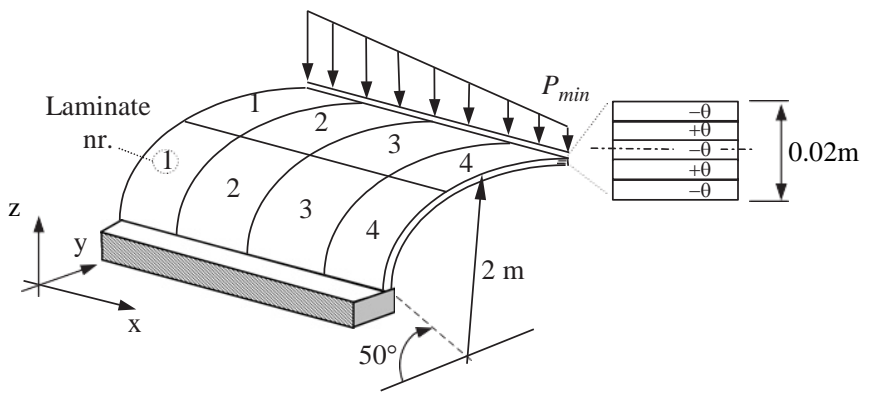

Fig. 1. Cylindrical shell and angle-ply composite laminates distribution (ply angle y referred to $x$-axis). 
Table 1

Mean values of mechanical properties of unidirectional composite layers

\begin{tabular}{|c|c|c|c|c|c|}
\hline Elastic constants & & Values & Mechanical strength & & Values \\
\hline \multirow[t]{3}{*}{ Elastic modulus (GPa) } & $E_{1}$ & 38.60 & Longitudinal (MPa) & & \\
\hline & $E_{2}$ & 8.27 & Tensile & $X$ & 1062 \\
\hline & & & Compression & $X^{\searrow}$ & 610 \\
\hline \multirow[t]{3}{*}{ Shear modulus (GPa) } & $G_{12}$ & 4.14 & Transversal (MPa) & & \\
\hline & $G_{13}$ & 4.14 & Tensile & $Y$ & 31 \\
\hline & $G_{23}$ & 4.14 & Compression & $Y^{A}$ & 118 \\
\hline \multirow[t]{3}{*}{ Poisson's coefficients } & $\mathrm{n}_{12}$ & 0.26 & & & \\
\hline & $n_{13}$ & 0.26 & Shear (MPa) & $S$ & 72 \\
\hline & $\mathrm{n}_{23}$ & 0.26 & & & \\
\hline
\end{tabular}

is a composite system of epoxy resin reinforced by unidirectional fibres of E Glass known as Scotchply 1002 with specific weight $\rho=1.8 \times 10^{3} \mathrm{~kg} / \mathrm{m}^{3}[17]$.

The vector of input parameters considered as nonstatistical correlated random variables of the structural system is $\mathbf{x}$ having the following components: longitudinal Young's modulus $E_{1, j}$, transversal modulus $E_{2, j}$, transversal tensile strength $Y_{j}$ and shear strength $S_{j}$, where the $j$ th index denotes the laminate number. These are the mechanical properties with the most critical deviations on the laminate strength randomness, according to the numerical simulation performed by António et al. [18]. Nevertheless, the presented study can be extended to other random variables. The number of physical properties considered as input parameters in the present analysis is sixteen: $E_{1, j}, E_{2, j}, Y_{j}$, $S_{j}, j=1, \ldots, 4$.

\subsection{System response functionals}

The stress analysis is performed using the strength parameter $R_{j}$ known as Tsai number and calculated as the ratio between the failure (or maximum allowable) stress and the actual stress at the $j$ th point of the structure where the stress vector is evaluated. The Tsai number $R_{j}$ is a function of the actual stresses and it is obtained by solving the interactive quadratic failure criterion of Tsai-Wu [17],

$\left(F_{\text {ik }} s_{i} S_{k}\right) R_{j}^{2}+\left(F_{i} s_{i}\right) R_{j}=1 \quad i, k=1,2,6$,

where $s_{i}$ is the ith component of the stress vector, $F_{\mathbb{l k}}$ and $F_{i}$ are strength parameters associated with unidirectional reinforced laminate defined from the macro-mechanical point of view [17].

In this work, two functionals are considered in the sensitivity-uncertainty analysis as presented in Section 3, one related with the maximum displacement on the structure,

$\bar{u}=\operatorname{Max}\left(u_{1}, \ldots, u_{r}\right), \quad r=1, \ldots, N_{\mathrm{dis}}$

and the second one related with the most critical Tsai number,

$\bar{R}=\operatorname{Max}\left(R_{1}, \ldots, R_{j}\right), \quad j=1, \ldots, N_{\text {str }}$
Table 2

Composite laminate distribution on structure used in sensitivity analysis

\begin{tabular}{ll}
\hline Laminate nr. & Stacking sequence \\
\hline 1 & {$[-75 /+75 /-75 /+75 /-75]$} \\
2 & {$[+25 /-25 /+25 /-25 /+25]$} \\
3 & {$[-75 /+75 /-75 /+75 /-75]$} \\
4 & {$[+25 /-25 /+25 /-25 /+25]$} \\
\hline
\end{tabular}

being $N_{\text {dis }}$ the total number of displacements and $N_{\text {str }}$ the total number of points where the stress vector is evaluated. Then, Eq. (11) is rewritten as

$\varphi=(a, \bar{R})$

that is the response vector of the structural system considered in the present analysis.

\subsection{Sensitivity analysis validation}

The validation of the sensitivity model presented in Section 4 is implemented using the cylindrical shell shown in Fig. 1. A set of discrete vertical loads varying from $P_{\max }=30 \mathrm{kN}$ to $P_{\min }=3.333 \mathrm{kN}$ is applied. The four laminates have the stacking sequence presented in Table 2 . The results obtained using the Adjoint Variable Method presented in Section 4 are compared with the ones obtained from the Forward Finite Difference Method and they are presented in Tables 3 and 4. A good agreement is reached using both approaches.

\subsection{Importance of input parameters using sensitivity analysis}

The objective of this section is to analyze the influence of input parameter deviations on the structural response. The geometrical definition and the laminate distribution of the example are the same used in sensitivity analysis validation.

The concept of relative sensitivity referred in Section 2 is defined as

$\frac{\partial \varphi^{*}}{\partial x_{i}}=\left|\frac{\partial \varphi}{\partial x_{i}}\right|\left|\frac{x_{i}^{0}}{\varphi^{0}}\right|$ 
and its analysis aims to compare the relative importance of input parameters on the response. The results obtained from the sensitivity analysis and using the concept of relative sensitivity are shown in Table 5 . The longitudinal Young's modulus $E_{1}$ is the most important input parameter when the structural response is analyzed using the maximum displacement $\bar{u}$. However, the transversal modulus $E_{2}$ and the transversal tensile strength $Y$ are more outstanding if the response is evaluated using the stress analysis through the Tsai number $\bar{R}$.

Table 3

Sensitivity of maximum displacement $a$ for mean values of input parameters AVM-Adjoint Variable Method; FDM-Finite Difference Method (increment: $0.1 \%$ of mean value)

\begin{tabular}{|c|c|c|}
\hline Sensitivity & AVM & FDM \\
\hline$\partial a / \partial E_{1,1}$ & $2.298 \times 10^{-12}$ & $2296 \times 10^{-12}$ \\
\hline$\partial u \bar{u} / \partial E_{1,2}$ & $3.711 \times 10^{-14}$ & $3.711 \times 10^{-14}$ \\
\hline$\partial \bar{u} / \partial E_{1,3}$ & $4.797 \times 10^{-14}$ & $4.797 \times 10^{-14}$ \\
\hline$\partial \bar{u} / \partial E_{14}$ & $-5.881 \times 10^{-15}$ & $-5.882 \times 10^{-15}$ \\
\hline$\partial a / \partial E_{21}$ & $2.762 \times 10^{-13}$ & $2760 \times 10^{-12}$ \\
\hline$\partial u / \partial E_{2,2}$ & $5.962 \times 10^{-13}$ & $5.962 \times 10^{-13}$ \\
\hline$\partial u / \partial E_{2,3}$ & $3.372 \times 10^{-14}$ & $3.370 \times 10^{-14}$ \\
\hline$\partial u ̈ / \partial E_{24}$ & $-2.369 \times 10^{-13}$ & $-2368 \times 10^{-12}$ \\
\hline
\end{tabular}

Table 4

Sensitivity of most critical Tsai number $\hat{k}$ for mean values of input parameters AVM-Adjoint Variable Method; FDM-Finite Difference Method (increment: $1 \%$ of mean value)

\begin{tabular}{lrr}
\hline Sensitivity & \multicolumn{1}{l}{ AVM } & \multicolumn{1}{l}{ FDM } \\
\hline$\partial K / \partial E_{1,1}$ & $1.331 \times 10^{-11}$ & $1.329 \times 10^{-11}$ \\
$\partial K / \partial E_{1,2}$ & $1.261 \times 10^{-12}$ & $1.261 \times 10^{-12}$ \\
$\partial K / \partial E_{1,3}$ & $6.431 \times 10^{-12}$ & $6.414 \times 10^{-12}$ \\
$\partial K / \partial E_{1,4}$ & $-6.148 \times 10^{-14}$ & $-6.221 \times 10^{-14}$ \\
$\partial K / \partial E_{2,1}$ & $2.641 \times 10^{-12}$ & $2.631 \times 10^{-12}$ \\
$\partial K / \partial E_{2,2}$ & $-1.064 \times 10^{-10}$ & $-1.054 \times 10^{-10}$ \\
$\partial K / \partial E_{2,3}$ & $9.667 \times 10^{-13}$ & $9.564 \times 10^{-13}$ \\
$\partial K / \partial E_{2,4}$ & $2.904 \times 10^{-12}$ & $2.890 \times 10^{-12}$ \\
$\partial K / \partial Y_{2}$ & $3.054 \times 10^{-18}$ & $3.052 \times 10^{-08}$ \\
$\partial K / \partial S_{2}$ & $9.375 \times 10^{-10}$ & $9.247 \times 10^{-10}$ \\
\hline
\end{tabular}

Other important concept in the present analysis is the coefficient of variation of the response taking an independent analysis for each input parameter. Using the sensitivity-uncertainty analysis of Section 3, namely Eqs. (6) and (10), the coefficient of variation is established as

$\mathrm{CV}_{\varphi}=\left(\frac{\sqrt{\operatorname{var}(\varphi)}}{|E(\varphi)|}\right)_{x}=\left|\frac{S_{i} \sigma_{i}}{\varphi^{0}}\right|$

being $\varphi^{0} \equiv \varphi\left(\mathbf{x}^{0}\right)$ and $S_{i}=\left(\partial \varphi / \partial x_{i}\right)_{x^{0}}$ the sensitivity of the response to parameter $x_{i}$, evaluated for mean values $\mathbf{x}^{0}=\left(x_{1}^{0}, \ldots, x_{n}^{0}\right)$. Considering the coefficient of variation of the input parameter $x_{i}$ defined as

$\mathrm{CV}_{x_{i}}=\left|\frac{\sigma_{i}}{x_{i}^{0}}\right|$

the relationship between the coefficient of variation of the response and the coefficient of variation of an input parameter is

$\mathrm{CV}_{\varphi}=\left|\frac{S_{i} \mathrm{CV}_{x_{x}} x_{i}^{0}}{\varphi^{0}}\right|=\frac{\partial \varphi^{*}}{\partial x_{i}} \mathrm{CV}_{x_{x}}$,

where $\partial \varphi^{*} / \partial x_{i}$ is the relative sensitivity defined in Eq. (30).

Fig. 2 shows the maximum values for the coefficient of variation of the response $\mathrm{CV}_{\varphi}$ considering $\mathrm{CV}_{x_{i}}=6 \%$ for the input parameters. The shape of the bar chart agrees with the conclusions obtained from the relative sensitivities presented in Table 5 .

The above analysis was performed considering an independent analysis for each input parameters in the equation of propagation of uncertainties (10). This analysis is important in order to evaluate the individual influence of each input parameter. However, the joint effects of the propagation of uncertainties on the response play an important role in structural reliability analysis.

The equation of propagation of uncertainties (15) is

$\mathrm{C}_{\mathrm{Q}}=\mathrm{SC}_{x} \mathrm{~S}^{\mathrm{T}}$.

If the input parameters are uncorrelated then matrix $\mathbf{C}_{x}$ is diagonal and taking $\mathrm{CV}_{x}=6 \%$ for all parameters, the

Table 5

Relative sensitivities

\begin{tabular}{|c|c|c|c|c|}
\hline \multirow[t]{2}{*}{ Sensitivity } & \multicolumn{4}{|c|}{ Macro-element/composite laminate } \\
\hline & $j=1$ & $j=2$ & $j=3$ & $j=4$ \\
\hline$\partial \vec{u}^{*} / \partial E_{1, j}$ & $8.228 \times 10^{-01}$ & $1.329 \times 10^{-\infty}$ & $1.718 \times 10^{-02}$ & $2106 \times 10^{-63}$ \\
\hline$\partial \hat{K}^{*} / \partial E_{1, j}$ & $5.252 \times 10^{-01}$ & $4.975 \times 10^{-62}$ & $2.537 \times 10^{-01}$ & $2426 \times 10^{-63}$ \\
\hline$\partial \vec{u} / \partial E_{2 j}$ & $2.119 \times 10^{-0.2}$ & $4.574 \times 10^{-60}$ & $2.587 \times 10^{-03}$ & $1.817 \times 10^{-0}$ \\
\hline$\partial \hat{R}^{*} / \partial E_{2 j}$ & $2.233 \times 10^{-0.2}$ & $8.994 \times 10^{-06}$ & $8.172 \times 10^{-03}$ & $2455 \times 10^{-e}$ \\
\hline$\partial \hat{K}^{*} / \partial Y_{j}$ & 0 & $9.677 \times 10^{-06}$ & 0 & 0 \\
\hline$\partial \hat{R}^{*} / \partial S_{j}$ & 0 & $6.900 \times 10^{-60}$ & 0 & 0 \\
\hline
\end{tabular}


above equation gives

$$
\begin{aligned}
\mathrm{C}_{\boldsymbol{\varphi}} & =\left[\begin{array}{cc}
\operatorname{var}(\bar{u}) & \operatorname{cov}(\bar{u}, \bar{R}) \\
\operatorname{cov}(\bar{u}, \bar{R}) & \operatorname{var}(\bar{R})
\end{array}\right] \\
& =\left[\begin{array}{cc}
2.846 E-05 & 1.504 E-04 \\
1.504 E-04 & 7.212 E-03
\end{array}\right] .
\end{aligned}
$$

The coefficient of variation of the response for each functional defined in (29) is as follows

$\mathrm{C} \quad \mathrm{CV}_{\tilde{u}}=\frac{\sqrt{\operatorname{var}(\bar{u})}}{|E(\bar{u})|}=\frac{\sqrt{\operatorname{var}(\tilde{u})}}{|\bar{u}|}$,

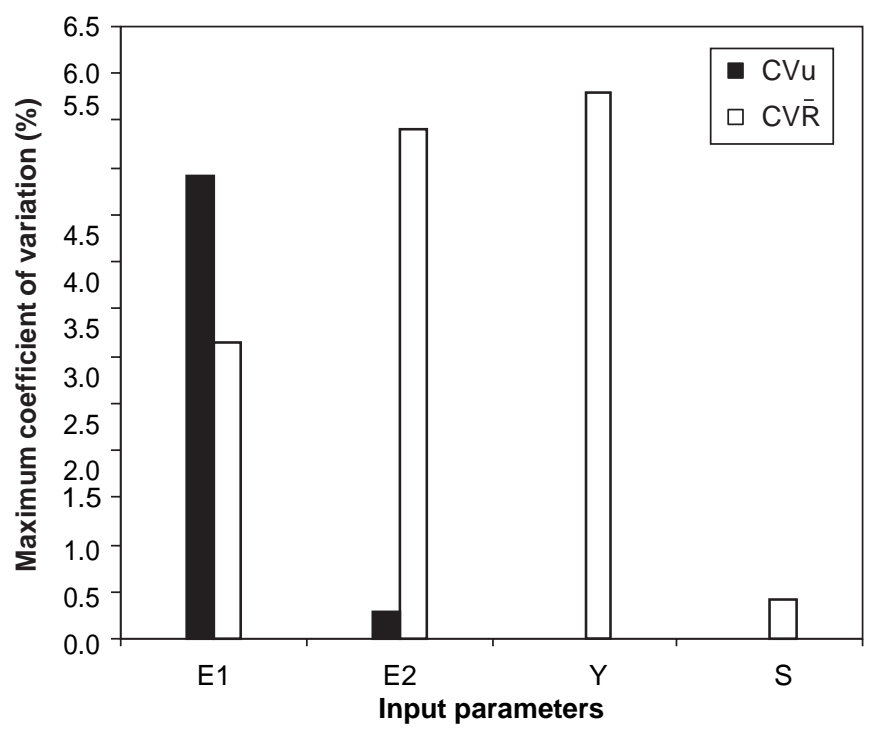

Fig. 2. Maximum values of the coefficients of variation $\mathrm{CV}_{\bar{u}} \partial \% \mathrm{p}$ and $\mathrm{CV}_{R^{0}} \partial \%$ for response functional. Independent analysis using $\mathrm{CV}_{x} \frac{1 / 4}{4} 6 \%$ for the input parameters.
$\mathrm{CV}_{\bar{R}}=\frac{\sqrt{\operatorname{var}(\bar{R})}}{|E(\bar{R})|}=\frac{\sqrt{\operatorname{var}(\bar{R})}}{\bar{R}}$.

In this example the mean values for structural response are $\bar{u}=0.1078 \mathrm{~m}$ and $\bar{R}=0.978$.

Using the covariance matrix (34) and the definitions (35) and (36) the following coefficients of variation of the response are obtained:

$\mathrm{CV}_{\bar{u}}=4.95 \%$ and $\quad \mathrm{CV}_{\bar{R}}=8.68 \%$.

\subsection{Propagation of uncertainties in angle-ply laminates}

In this section the influence of anisotropy in uncertainties propagation is studied. An example with balanced $\pm \theta$ angle-ply laminates with five layers as shown in Fig. 1 has been implemented. The vertical loads vary linearly from $P_{\max }=7.500 \mathrm{kN}$ to $P_{\min }=0.833 \mathrm{kN}$. The ply angle $\theta$ is referred to $x$-axis as shown in Fig. 1 .

Fig. 3 presents the behavior of critical displacement $\bar{u}$ and critical Tsai number $\vec{R}$ when the ply angle varies from $0^{\circ}$ to $90^{\circ}$.

The critical displacement increases from a minimum value at $0^{\circ}$ reaching at $90^{\circ}$ a value corresponding to four times that minimum. The critical Tsai number increases six times from a minimum value along the domain of $\theta$. This shows the important influence of anisotropy with consequences on uncertainties propagation.

To study the influence of uncertainties propagation on the response a coefficient of variation $\mathrm{CV}_{x}=6 \%$ is considered for the input parameters. The analysis is implemented independently for each input parameter using Eqs. (31)-(33). Figs. 4 and 5 show this analysis results using the maximum coefficient of variation for the critical displacement $a$ and the critical Tsai number $\bar{R}$ presented as functions of ply angle $\theta$.

The maximum coefficient of variation of the critical displacement, $\mathrm{CV}_{\bar{u}}(\%)$ presents a quasi-symmetric beha-

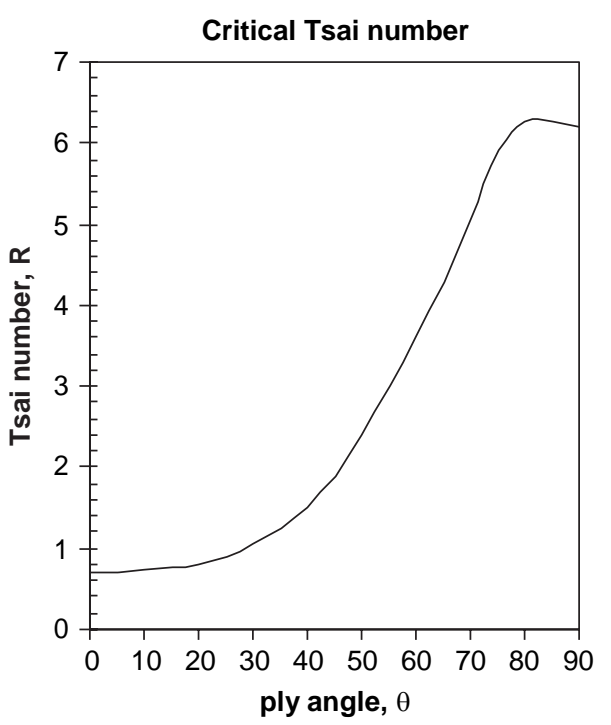

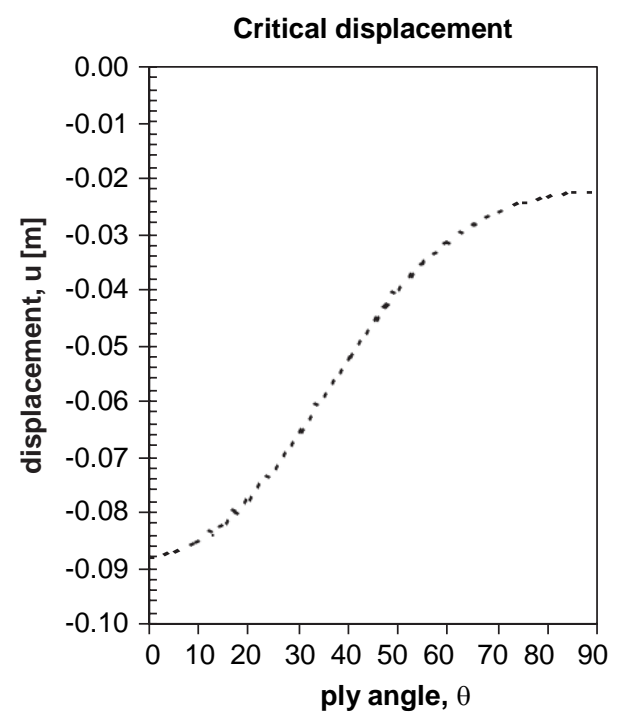

Fig. 3. Influence of anisotropy (ply angle y) on response functional $\bar{u}$ and $\bar{R}$. 
vior comparing both input parameters the longitudinal Young's modulus $E_{1}$ and the transversal modulus $E_{2}$ as shown in Fig. 4. The maximum coefficient of variation of the critical Tsai number, $\mathrm{CV}_{R} ð \%$ p presents the highest values at y $1 / 4551$ for $E_{2}$ and at y $1 / 451$ for $E_{1}$ as shown in Fig. 5. The same figure shows that uncertainties in

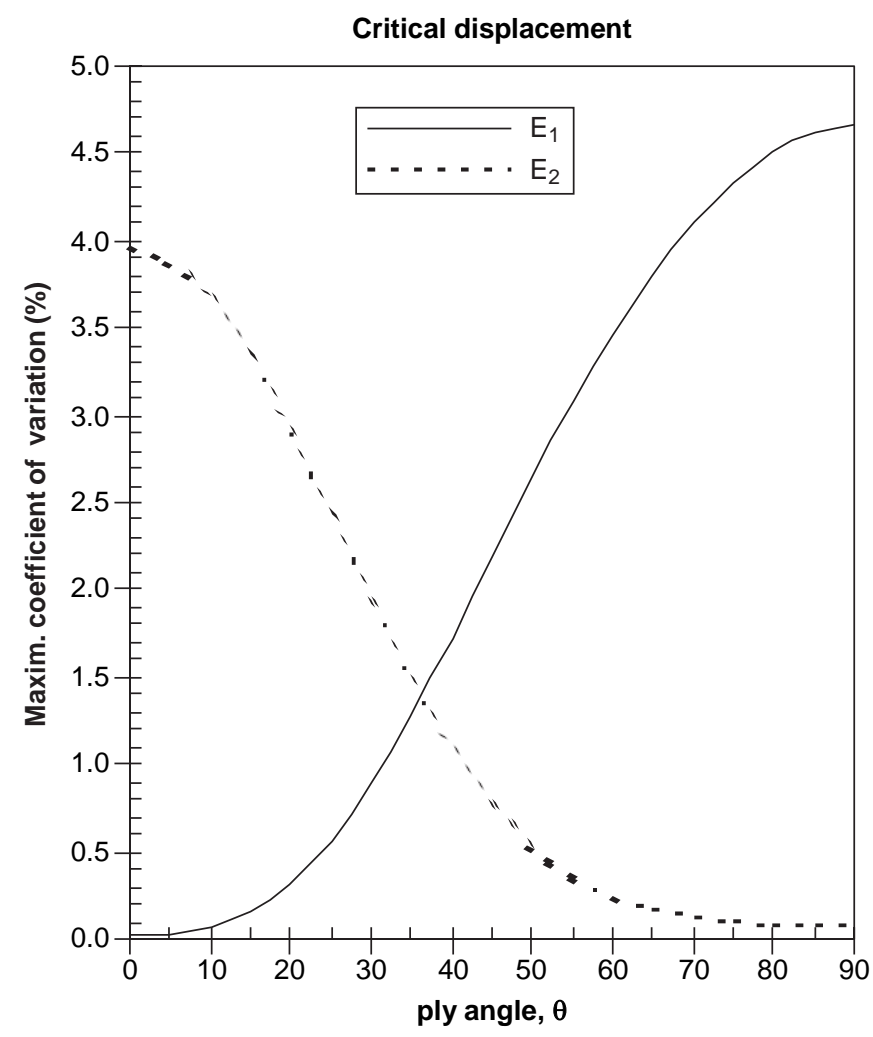

Fig. 4. Maximum coefficient of variation of the critical displacement, $\mathrm{CV}_{u^{-}}$ ¡\% p. Independent analysis using $\mathrm{CV}_{x} 1 / 46 \%$ for the input parameters $E_{1, j}$ and $E_{2, j}, j 1 / 41, \mathbf{Y}, 4$. transversal tensile strength $Y$ have large influence on uncertainties of the response for most of the values of the domain of $y$. In opposite way uncertainties in shear strength $S$ propagates fairly on the response.

Using the covariance matrix (15) and the definitions (35) and (36) it is possible to analyze the joint effects of all input parameters for the uncertainties propagation on the response. Fig. 6 presents the behaviors of the coefficients of variations $\mathrm{CV}_{\bar{u}} ð \% \mathrm{P}$ and $\mathrm{CV}_{\bar{R}^{\partial}}$ \% $\mathrm{P}$ as functions of ply angle $y$.

The behavior of displacement coefficient of response $\mathrm{CV}_{\bar{u}} \% \% \mathrm{p}$ is dominated by the maximum coefficient of variation when comparing Figs. 4 and 6 . In this case there are no synergetic effects in uncertainties propagation on response. However, the comparison of Figs. 5 and 6 shows an important synergetic effect on the coefficient of variation for Tsai number $\mathrm{CV}_{\bar{R}^{\gamma}}$ \% p. Indeed, this coefficient of variation of the response obtained from the joint analysis established by Eq. (15) and presented in Fig. 6 is always higher than the maximum coefficient of variation obtained using an independent analysis as shown in Fig. 5. The amplitude of the synergetic effect depends on ply angle $\mathrm{y}$ of the composite laminate. Since the Tsai number is related with the structural failure the referred synergetic effect on uncertainties propagation assumes an important role in structural reliability analysis.

Figs. 7 and 8 show the behavior of the coefficient of variation of the response with variations in ply angle $y$ for different coefficients of variation of the input parameters. Fig. 7 shows that the uncertainties propagation is mild for critical displacement response functional. On other hand, the synergetic effect on the coefficient of variation for Tsai number is amplified when increasing the coefficients of variation of the input parameters becoming very important for high uncertainty amplitudes.
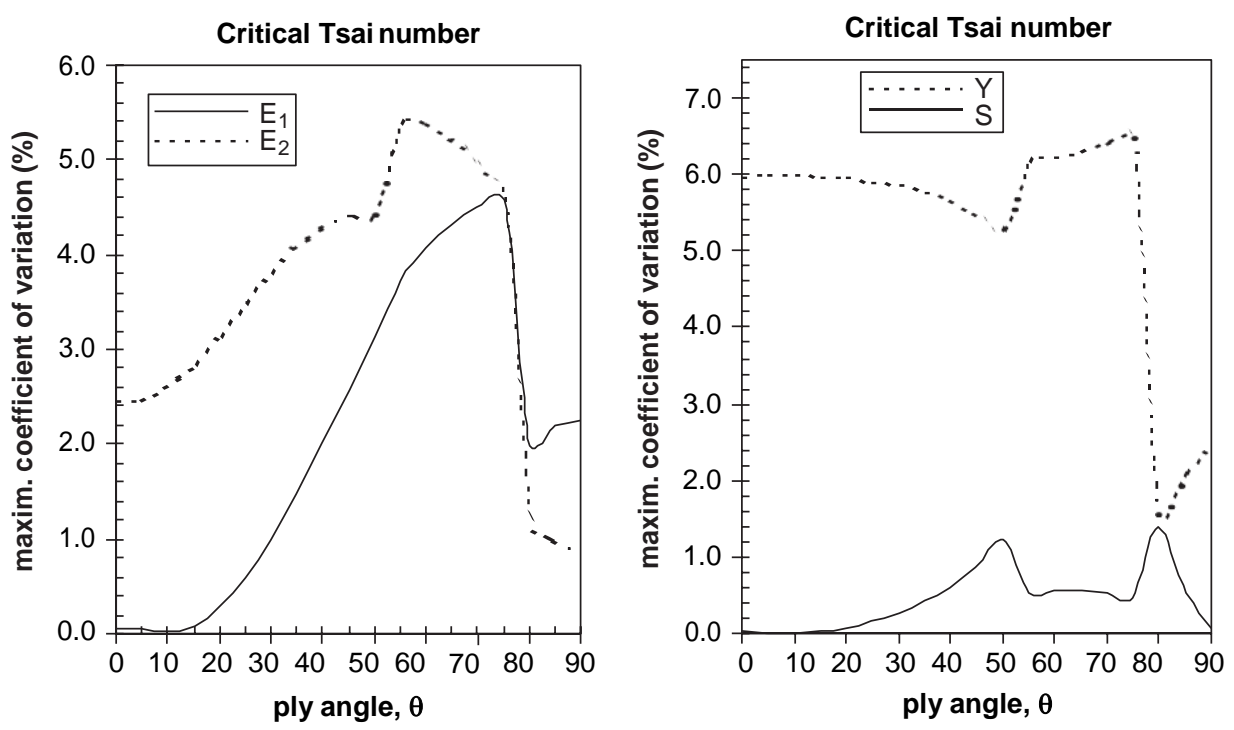

Fig. 5. Maximum coefficient of variation of the critical Tsai number, $\mathrm{CV}_{\tilde{R}^{\gamma}} \%$ p. Independent analysis using $\mathrm{CV}_{x}{ }^{1 / 4} 6 \%$ for the input parameters $E_{1, j}, E_{2, j}$, $Y_{j}, S_{j}, j^{1 / 4} 1, \mathrm{Y}, 4$. 


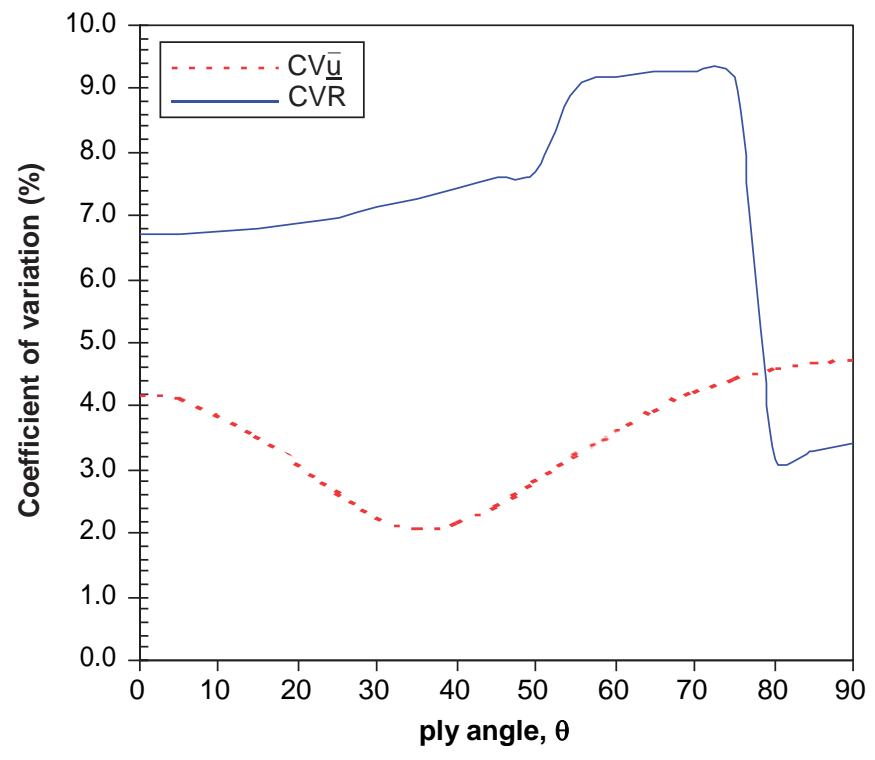

Fig. 6. Coefficient of variation of response $\mathrm{CV}_{\vec{u}} \gamma \% \mathrm{p}$ and $\mathrm{CV}_{R^{0}} \gamma \% \mathrm{p}$ using Eq. (15), $\mathrm{CV}_{x} 1 / 46 \%$ for the input parameters.

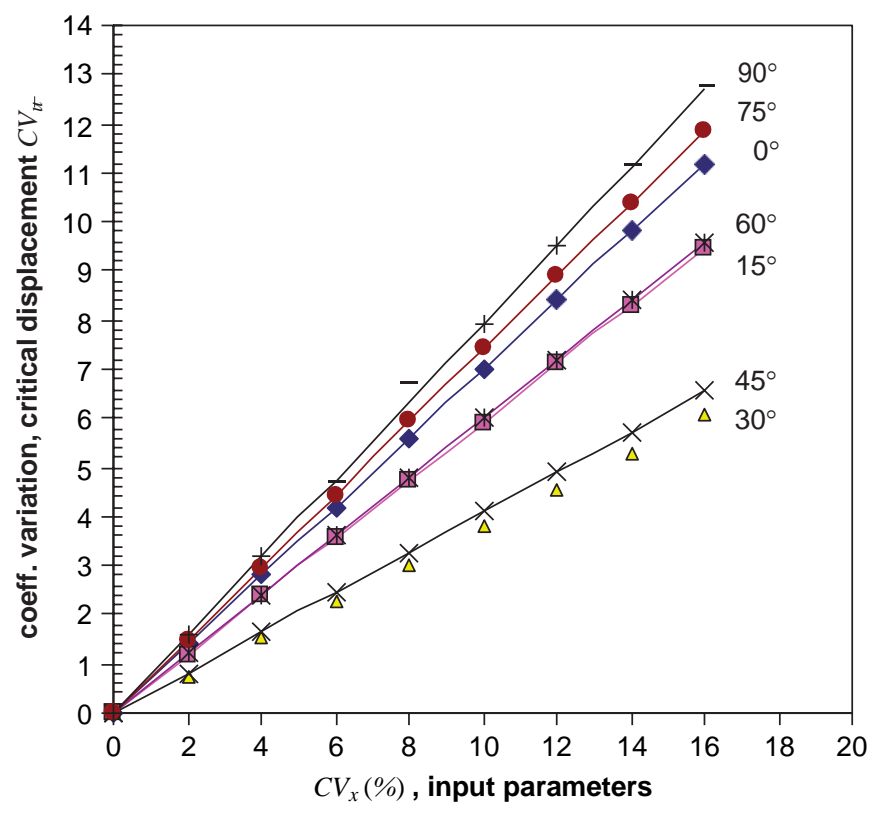

Fig. 7. Displacement coefficient of variation $\mathrm{CV}_{\vec{u}} \gamma \%$ p with variations in ply angle $\mathrm{yU}$ Input parameters changing simultaneously as defined in Eq. (15).

The methodology proposed by Oh and Librescu [13] can be applied to structural tailoring of angle-ply composite structures in the presence of input parameters uncertainties. From Fig. 3 reporting the influence of ply angle y over critical displacement and critical Tsai number, the most favorable values for ply angle belong to the interval [751, 901]. From Fig. 6 showing the behavior of the coefficients of variation of response $\mathrm{CV}_{\bar{u}} ð \% \mathrm{p}$ and $\mathrm{CV}_{\bar{R}} ð \% \mathrm{p}$ with ply angle $y$, the most favorable value aiming the mitigation of uncertainty propagation on structural response is $y / 4801$. This is the value that will be considered in structural

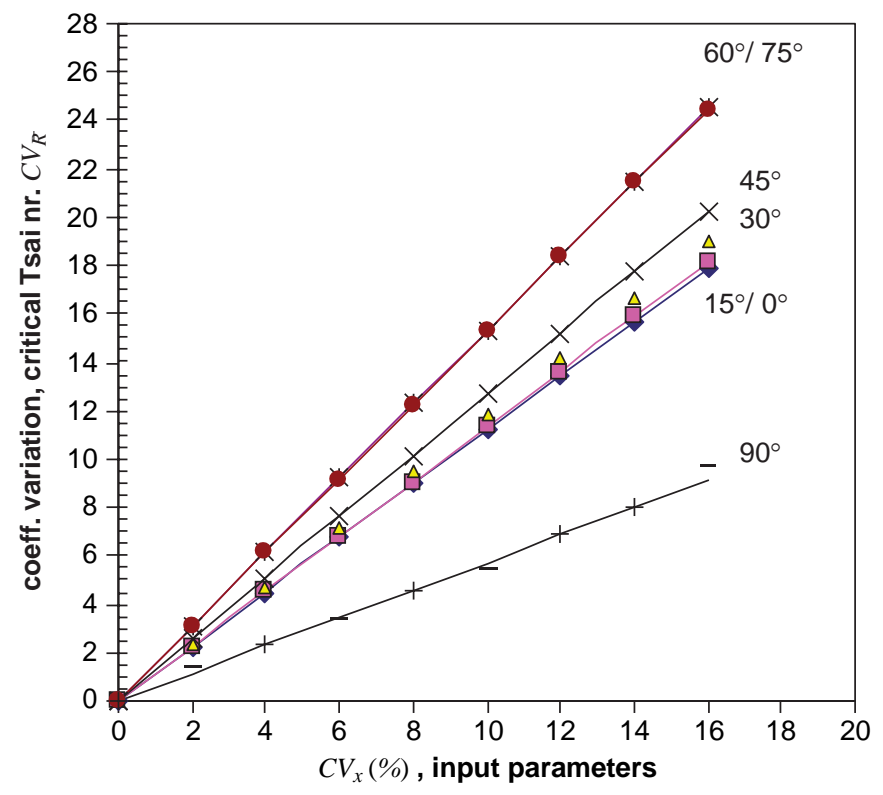

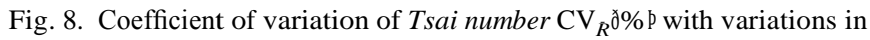
ply angle $\mathrm{yU}$ Input parameters changing simultaneously as defined in Eq. (15).

tailoring of angle-ply composite structures according to the combined analysis of Figs. 3 and 6.

\section{Conclusions}

The sensitivity-uncertainty analysis for composite structures presented here exhibits three major advantages: (1) important input parameters are identified; (2) the evaluation of the response uncertainty is done in a simple, systematic way; and (3) any changes on the uncertainties of the input parameters are easily incorporated in order to obtain the new uncertainties propagation on response.

The case study presented for angle-ply laminate composites reveals an important synergetic effect in uncertainties propagation for Tsai number associated with stress response of the composite structures. This synergetic effect must be considered as an important factor in reliability based design of angle-ply composite structures.

The structural tailoring technique based on the proposed sensitivity-uncertainty analysis is very useful in designing laminated composite structures minimizing the unavoidable effects of the input parameter uncertainties on structural reliability.

\section{References}

[1] Helton JC. Uncertainty and sensitivity analysis techniques for use in performance assessment for radioactive waste disposal. Reliab Eng Syst Saf 1993;42(2-3):327-67.

[2] Frey HC, Patil SR. Identification and review of sensitivity analysis. Risk Anal 2002;22(3):553-78. 
[3] Cacuci DG, Ionescu-Bujor M. A comparative review of sensitivity and uncertainty analysis of large-scale systems-II: statistical methods. Nucl Sci Eng 2004;147(3):204-17.

[4] Helton JC, Davis FJ, Johnson JD. A comparison of uncertainty and sensitivity analysis results obtained with random and Latin hypercube sampling. Reliab Eng Syst Saf 2005;89(3):305-30.

[5] Ahammed M, Melchers RE. Gradient and parameter sensitivity estimation for systems evaluated using Monte Carlo analysis. Reliab Eng Syst Saf 2006;91(6):594-601.

[6] Wu Y-T, Mohanty S. Variable screening and ranking using samplingbased sensitivity measures. Reliab Eng Syst Saf 2006;91(6):634-47.

[7] Bae H-R, Grandhi RV, Canfield RA. Epistemic uncertainty quantification techniques including evidence theory for large-scale structures. Comput Struct 2005;82(13-14):1101-12.

[8] Frangopol DM, Maute K. Life-cycle reliability-based optimisation of civil and aerospace structures. Comput Struct 2003;81(7):397-410.

[9] Conceie ã Antóio CA, Torres Marques A, Gone alves JF. Reliability based design with a degradation model of laminated composite structures. Struct Optim 1996;12:16-28.

[10] Conceie ã Antóio CA. A hierarchical genetic algorithm for reliability based design of geometrically non-linear composite structures. Composite Struct 2001;54:37-47.
[11] Yang J, Liew KM, Kitipornchai S. Stochastic analysis of compositionally graded plates with randomness under static loading. Intern J Mech Sci 2005;47:1519-41.

[12] Walker M, Hamilton R. A technique for optimally designing fibrereinforced laminated plates with manufacturing uncertainties for maximum buckling strength. Eng Optimization 2005;37(2):135-44.

[13] Oh DH, Librescu L. Free vibration and reliability of composite cantilevers featuring uncertain properties. Reliab Eng Syst Saf 1997;56:265-72.

[14] Cacuci DG. Sensitivity and uncertainty analysis. Theory, vol. 1. Boca Raton, FL: Chapman \& Hall/CTC Press; 2003.

[15] Arora JS, Cardoso JB. Variational principle for shape design sensitivity analysis. AIAA J 1992;30:538-47.

[16] Ahmad S. Curved finite elements in the analysis of solid, shell and plate structures. $\mathrm{PhD}$ thesis. UK: University College of Swansea; 1969.

[17] Tsai SW. Composites design. Dayton, USA: Think Composites; 1987.

[18] Conceie ã Antóío CA, Torres Marques A, Soeiro AV. Influence of physical properties randomness in laminated composites strength. In Proceedings of ICCM-9. Madrid, Spain: University of Zaragoza \& Woodhead Publishing, Ltd; 1993. 